\title{
Comparing the Retentive Force of O-ring and Several Retained Systems for One-piece Root-form Implant-retained Overdentures
} Mariko Kobayashi, DDS

Department of Removable Prosthodontics, Tsurumi University School of Dental Medicine, Yokohama, Japan

\section{Clinical significance}

This study clarified the retentive forces of a new retention system for one-piece implant (Nobel Direct: ND)retained overdentures. Using a medium-sized O-ring, proper retention could be obtained. Within the angle of $12^{\circ}$ created by two NDs, the double NDs produced forces $1.5^{-}$to 2 -fold greater than those of the single ND.

\begin{abstract}
Purpose: Little is known about the connection between the Nobel Direct (ND; Nobel Biocare, Sweden) abutment head and the denture base when ND is used for an overdenture. This study evaluated the retentive force of several retainers for ND-retained overdentures.

Methods: The bodies of two sizes of ND [3.0-mm dia. (ND3.0); 4.3- $\mathrm{mm}$ dia. (ND4.3)] were embedded in a brass patrix frame $(10 \times 10 \times 25 \mathrm{~mm})$ by using autopolymerized polymethyl methacrylate (PMMA; Unifast II, GC Corp.). Four sizes of O-rings [O3 (1.6-mm dia.), O4 (1.9-mm dia.), O5 (2.8-mm dia.), O6 (3.8-mm dia.)], ND healing caps (HC; Nobel Biocare), and protection caps (PC; Straumann) were prepared for attachment matrixs. $\mathrm{O} 3$ to $\mathrm{O} 6, \mathrm{ND}$, and $\mathrm{PC}$ were placed at the ND3.0 and ND4.3 abutment heads, respectively. As a control, the PMMA resin (RE) was directly used to connect the abutment head to the denture base resin without retainers. For ND4.3, the entire and upperhalf of the abutment head was covered with the above materials to compare the different contact areas between the patrix and matrix. The connected patrix and matrix frames were mounted on a screw-driven mechanical testing machine (Model UTM II, Toyo Boldwin, Japan). The retention of double retainers (20-mm apart) were also measured with the angles of two ND4.3 abutment heads of 0, 6, and 12 degrees. The retentive force $(n=5, N)$ obtained at a crosshead speed of $40 \mathrm{~mm} / \mathrm{min}$ was analyzed by analysis of vari-
\end{abstract}

\footnotetext{
Corresponding to: Dr Mariko Kobayashi Department of Removable Prosthodontics, Tsurumi University School of Dental Medicine, 2-1-3 Tsurumi, Tsurumi-ku, Yokohama, Kanagawa 230-8501, Japan

Tel: +81-45-581-1001, Fax: +81-45-573-9599

E-mail:masamari@agate.plala.or.jp
}

Received on May 25, 2006 / Accepted on September 5, 2006 ance (ANOVA)/Scheffé's test $(\alpha=0.05)$.

Results: The retentive force of ND3.0 and ND4.3 was significantly the greatest when $\mathrm{O} 3$ and $\mathrm{PC}$, respectively, $(P<0.05)$ were used. For ND3.0, the retentive force of $\mathrm{O} 4, \mathrm{RE}$, and $\mathrm{HC}$ was one-third of that of $\mathrm{O} 3$. For ND4.3, the retentive force of RE and O5 was twothirds of that of PC and that of $\mathrm{O} 6$ was one-third of the retentive force of PC. The retentive force of full contact was approximately two times greater than that of the upper-half contact. The angle of the two NDs did not affect the retentive force $(P>0.05)$.

Conclusion: When the medium-sized O-ring, PMMA, and $\mathrm{HC}$ were used, an appropriate retentive force was obtained for the ND implant-retained overdentures.

Key words: one-piece implant, implant-retained overdenture, O-ring, retentive force, Nobel Direct

\section{Introduction}

For approximately 15 years, immediate loading or early loading has been clinically attempted. ${ }^{1-8}$ Recently, the Nobel Direct (ND; Nobel Biocare, Sweden)-a one-piece tapered implant-was developed for immediate loading. As shown in Figure 1, ND is composed of an abutment head (5-mm long), shoulder (3- $\mathrm{mm}$ long), and body (3.0- $\mathrm{mm}$ dia., 10-, 13-', and 15-mm long; 3.5-, 4.3-, 5.0-mm dia., 10-, 13-', 16-mm long). The ND abutment head has a conical shape with six degrees of taper, and the implant body is processed to a rough surface (TiUnite). ${ }^{9-12}$

Since ND placement can allow flapless surgery, the surgical time is shortened, and minimally invasive surgery mitigates postoperative swelling and pain. Furthermore, the tapered body and the TiUnite surface provide immediate function and soft tissue integration. Although there are no references on the stress analysis of ND, the survival rate of ND was similar to that of conventional implants. ${ }^{3}$

Because NDs are one-piece implants, abutment selection is not necessary. If NDs were placed without parallel or few crealance to op- 
posite teeth, the abutment head can be cut by using the system's carbide burs. ${ }^{10-12}$ Most of the superstructures for NDs are cemented to the abutment head. However, there are no manufacturer's instructions for ND-retained overdentures, particularly regarding retainer selection and fabrication. Clinically, the intaglio surface of an overdenture is adjusted using autopolymerized polymethyl methacrylate (PMMA) resin in order to fit the abutment head of the NDs. Alternatively, a rubber O-ring can be fitted on the implant head to obtain the retentive force for the ND-retained overdenture (Figs. 2a, b). Although there were no experimental data, O-rings were mainly used in our practice, and sufficient retention was obtained..$^{11,12,15,16}$ If the appropriate bone shape is correctly confirmed by computerized tomography (CT) beforehand, the NDs can be placed with flapless surgery by using the existing complete denture by drilling holes. After placement, the existing denture can be immediately modified to the ND-retained overdenture by using the overlay technique. ${ }^{12}$ Surprisingly quick improvement in denture stability is obtained for the patient. Few clinical reports indicated that ball attachment (Nobel Biocare) was used for the ND-retained overdentures. ${ }^{12}$ However, this retention system cannot be applied to many cases because a large amount of denture inter-ridge space is necessary.

Transitional implants (TIs) have been recently used to support provisional restorations during healing periods. ${ }^{13,14}$ To increase denture stability, the existing complete dentures were also immediately modified to TI-retained overdentures. In a previous study, the retentive force of the O-ring attachment to one of the TIs, i.e., the Immediate Provisional Implant (IPI; Nobel Biocare), was measured in vitro. ${ }^{15}$ The use of smaller O-rings was recommended for IPI-retained overdentures. ${ }^{16}$ However, little attention has been given to the retention system for ND-retained overdentures, particularly the retentive force of NDs.

The purpose of this study was to investigate the retention of ND-retained overdentures by using several retention systems. The in vitro retentive force of the O-rings and other materials attached to ND abutment heads was measured for both single retainers by using one ND and double retainers by using two NDs.

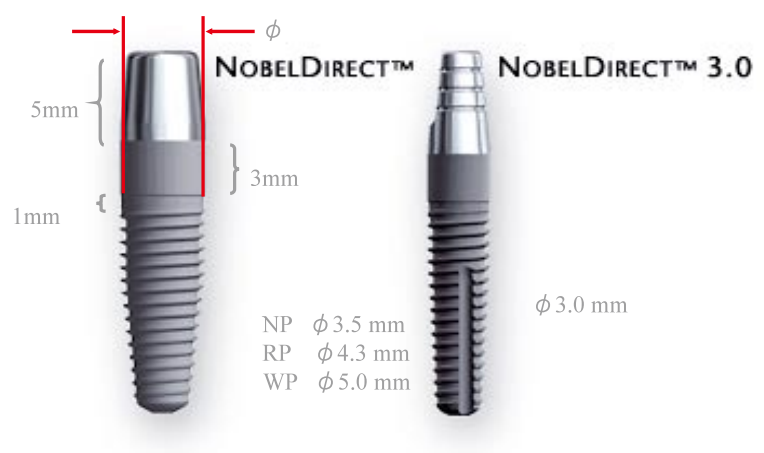

Fig. 1 Diagram of the NOBEL DIRECT.

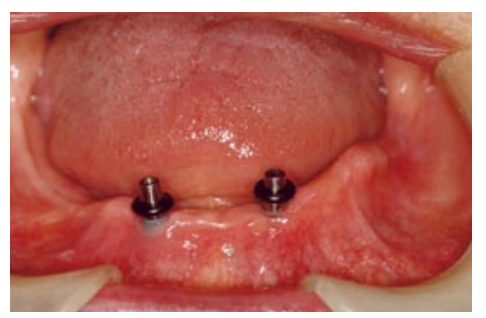

$\mathbf{a}$

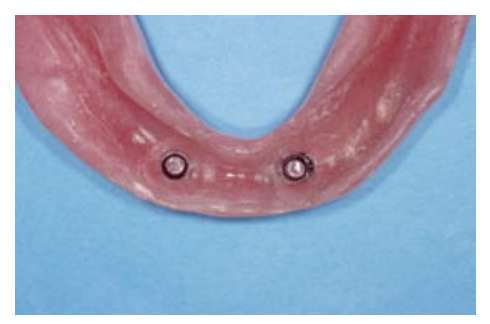

b

Fig. 2 Rubber O-ring used for the ND-retained overdenture.

\section{Materials and methods}

\section{Specimen preparation}

This study measured the retentive force of one ND (single retainer) and two NDs (double retainer).

To test the single retainer, upper and lower cylindrical-shaped frameworks $(10 \times 10 \times 25 \mathrm{~mm})$ were milled with brass alloy so that both frameworks were properly fitted as a joined patrix and matrix assembly (Fig. 3). Since two lower frameworks were commonly used, 65 upper frameworks were prepared to fit them. Each ND3.0 (3.0-mm dia.) and ND4.3 (4.3 $\mathrm{mm}$ dia.) body was embedded to shoulder level in the lower framework with the PMMA resin (Unifast II, GC Dental Industrial Corp., Tokyo, Japan). The hollow in all the upper frameworks was also filled with the PMMA resin. After the PMMA in the lower 


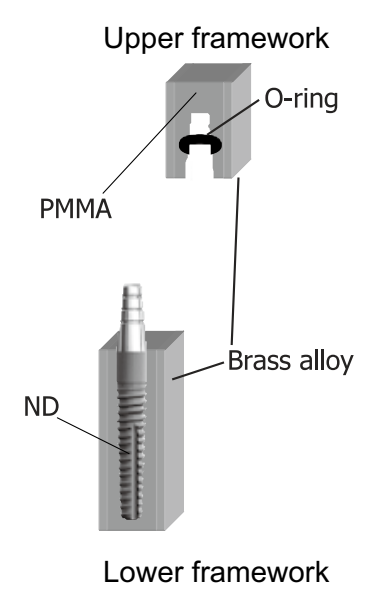

Fig. 3 Upper and lower cylindrical-shaped frameworks were milled with brass alloy so that both frameworks properly fit as patrix and matrix joined to make a single retainer.

framework was completely polymerized, a resin separator (petroleum jelly) was placed on the connecting surface of the PMMA. For ND3.0, three sizes of O-rings [Acrylonitrile-butadiene rubber (NBR); No. 3 (O3) and No. 4 (O4), Inoue attachment, Tokyo; No. 5 (O5), SAN-EI, Osaka, Japan] were placed at the bottom level of the abutment head. The inner and outer diameters of the O-rings used in this study are shown in the Table 1. For ND4.3, two sizes of O-rings [No. 5 (O5) and No. 6 (O6), SAN-EI (Table 1)] were placed as in the case of ND3.0. Some PMMA resin was removed from the upper framework to accommodate the $\mathrm{O}$-ring on top of the abutment head. After the upper framework was securely fitted to the lower framework with the proper fit of the patrix and matrix, PMMA was overlaid in the hollow of the upper framework. Similarly, two healing caps (HC, Nobel Biocare and protection caps (PC), Straumann) were placed on the ND3.0 and ND4.3 abutment heads, respectively, and then they were fixed in the upper framework by using PMMA. As a control, PMMA (RE, Unifast II, GC Dental Industrial Corp.) was used to connect the abutment head to the hollow of the PMMA in the upper framework without an O-ring and a healing cap. After polymerization, the excess resin was trimmed. For ND4.3, specimens were fabricated in which only the upper half of the abutment head was covered with the above materials to compare the different contact areas between the patrix and matrix. For clinical situations in which the ND was deeply placed, the O-ring was placed at $5 \mathrm{~mm}$ above the junction between the machine surface and rough sur-
Table 1 Inner and outer diameter of O-rings (O3 to O6) used in this study.

\begin{tabular}{|c|c|c|c|c|}
\hline & Inner & Outer & & \\
\hline & Diameter(mm) & Diameter $(\mathrm{mm})$ & Width $(m m)$ & Manufacturer \\
\hline $\mathrm{O} 3$ & 1.6 & 5.0 & 1.7 & \multirow{2}{*}{$\begin{array}{c}\text { Inoue } \\
\text { Attachment }\end{array}$} \\
\hline $\mathrm{O} 4$ & 1.9 & 5.5 & 1.8 & \\
\hline $\mathrm{O} 5$ & 2.8 & 6.6 & 1.9 & \multirow[b]{2}{*}{ SAN-EI } \\
\hline $\mathrm{O} 6$ & 3.8 & 7.6 & 1.9 & \\
\hline
\end{tabular}

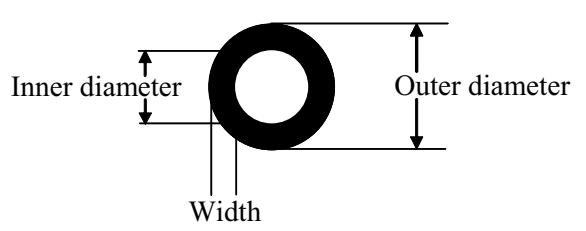

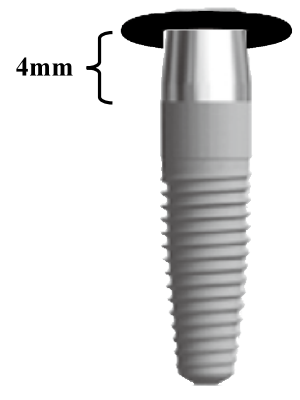

Single (Upper)

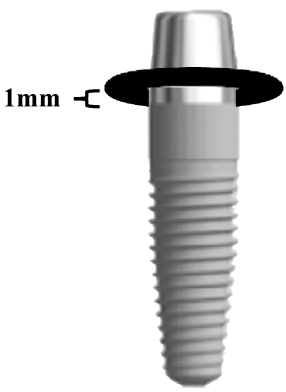

Single (Normal)
Fig. 4 The different contact areas between the patrix and matrix of ND4.3.

face as shown in Figure 4. Five matrix specimens were prepared for each retention type; a total of 65 specimens were fabricated $(n=5,25$ specimens for ND3.0 and 40 specimens for ND4.3).

For the double retainer, upper and lower acrylic blocks of the same size $(15 \times 30 \times 50 \mathrm{~mm})$ were prepared to assess the retentive force of ND4.3. Two ND4.3 implants were placed $20 \mathrm{~mm}$ apart in the lower blocks, according to in vivo procedures. The angles of both the ND4.3 abutment heads were designed to be parallel, 6 , and $12 \mathrm{de}$ grees (Fig. 5). The angles of each specimen were confirmed using a profile projector $(\times 50 ; \mathrm{V}-16 \mathrm{E}$, Nikon, Japan). Next, the upper blocks were drilled to adapt the O-ring on top of the abutment head. Similar to the single retainer, the $\mathrm{O}$-rings and $\mathrm{HC}$ were placed at the bottom level of the ND4.3 abutment head. After the upper and lower blocks were securely fitted, PMMA was 


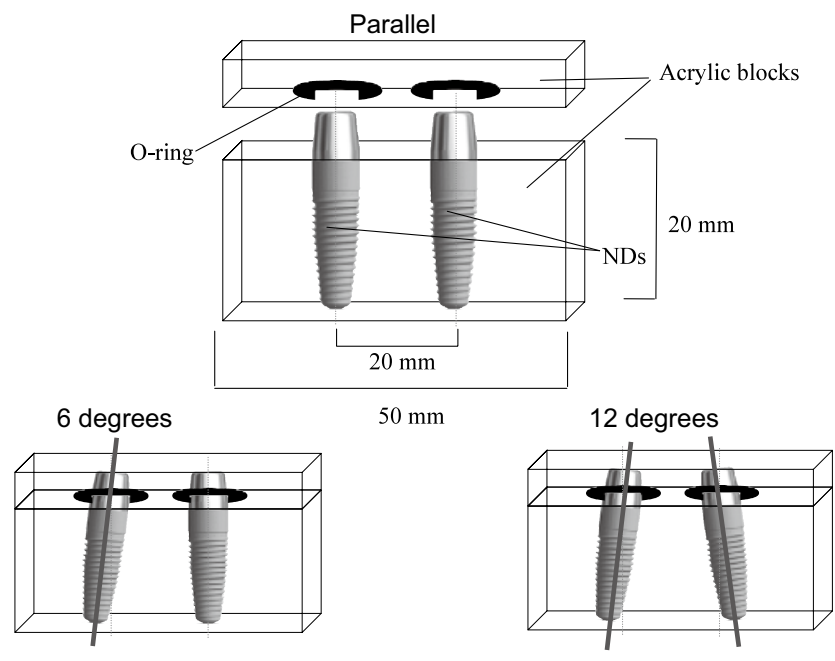

Fig. 5 The angle conditions of the two ND4.3 abutment heads were designed as parallel, 6, and 12 degrees for a double retainer.

overlaid in the hollow of the upper framework. Twenty matrix specimens were prepared for each of the three patrix specimens with different angles ( $n=5$, total 60 specimens).

\section{Measurement of retentive force}

All the specimens were soaked in distilled water for more than one week before testing. Using a constant load-press machine (Seiki, Tokyo), the upper and lower frameworks and acrylic blocks were joined under a 2 -kg force. The joined frameworks and blocks were mounted on a screwdriven mechanical testing machine (Model UTM II, Tokyo Boldwin, Japan). The retentive force (N) was evaluated as the tensile force obtained when the upper and lower frameworks or blocks were separated at a crosshead speed of $40 \mathrm{~mm} / \mathrm{min}$. This measurement was repeated five times for each test specimen. The data were analyzed using the SPSS statistical package (Version 10.0, SPSS Inc., Chicago, IL) by one-way analysis of variance (ANOVA) and Scheffe's multiple comparison test at a significance level of $\alpha=0.05$.

\section{Results}

The retentive force of the ND3.0 and ND4.3 single retainers is shown in Figures 6 and 7, respectively. The retentive force of the ND3.0 retainer for all materials ranged from 1.56 to $19.5 \mathrm{~N}$. The retentive force of $\mathrm{O} 3$ was significantly $(P<0.05)$ the greatest among all the materials tested.

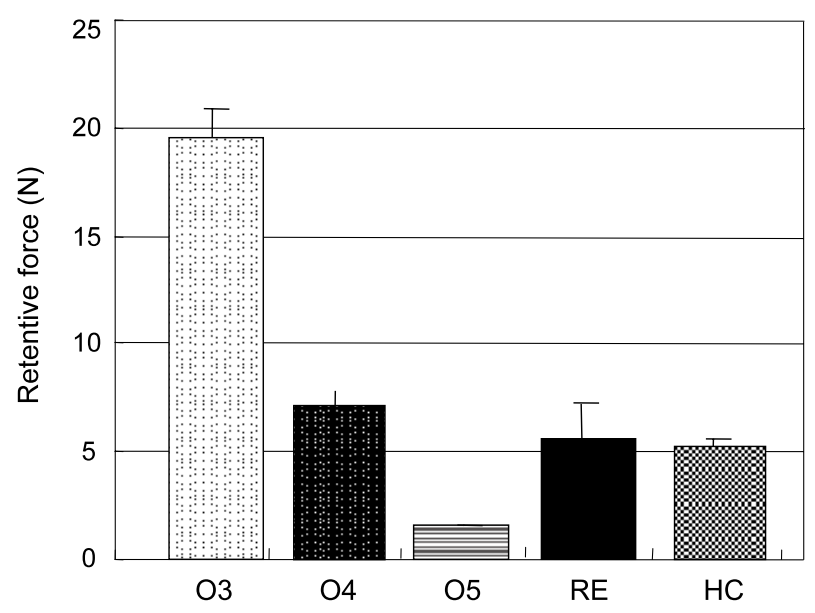

Fig. 6 Retentive force of the ND3.0 single retainer. O3: O-ring, 1.6-mm inner diam., O4: O-ring, 1.9-mm diam., O5: O-ring, 2.8-mm diam, RE: Auto polymerized acrylic resin, HC: Healing cap.

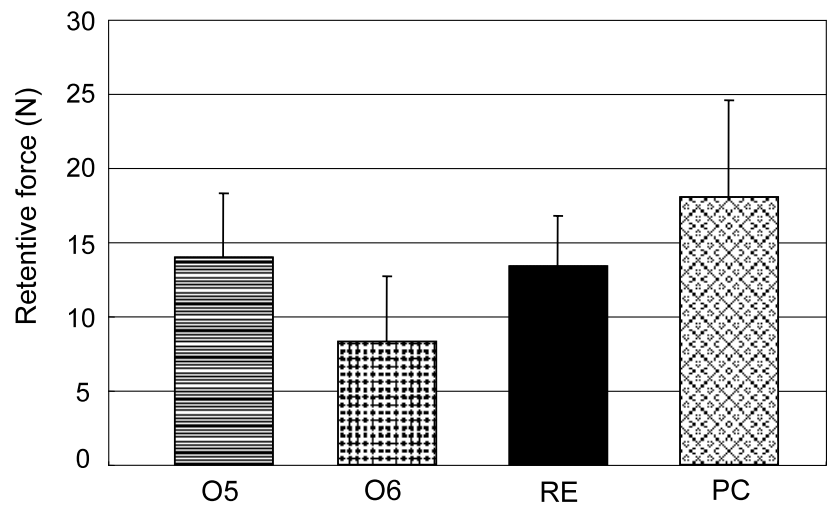

Fig. 7 Retentive force of an ND4.3 single retainer. O5: O-ring 2.8-mm diam., O6: O-ring 3.8- $\mathrm{mm}$ diam., RE: Autopolymerized acrylic resin, PC: Protection cap.

There were no significant differences $(P>0.05)$ in retentive force among $\mathrm{O} 4, \mathrm{RE}$, and $\mathrm{HC}$. The values of the retentive force for $\mathrm{O} 4, \mathrm{RE}$, and $\mathrm{HC}$ were almost equivalent (approximately 5.2 to 7.09 $\mathrm{N})$ and about four times greater than those for O5. Of the O-rings, the largest one exhibited the smallest retentive force.

With regard to all the materials tested for $\mathrm{ND} 4.3$, the retentive force ranged from $8.27 \mathrm{~N}$ to $18.1 \mathrm{~N}$. The retentive force was significantly the highest for $\mathrm{PC}$, whereas it was the lowest for $\mathrm{O} 6$ $(P<0.05)$. There were no statistical differences in retentive force between $\mathrm{O} 5$ and $\mathrm{RE}(P>0.05)$. Similar to ND3.0, the retentive force for the larger O-ring was approximately 2.5 times higher than that for the smaller one.

Figure 8 shows the retentive force of the dou- 


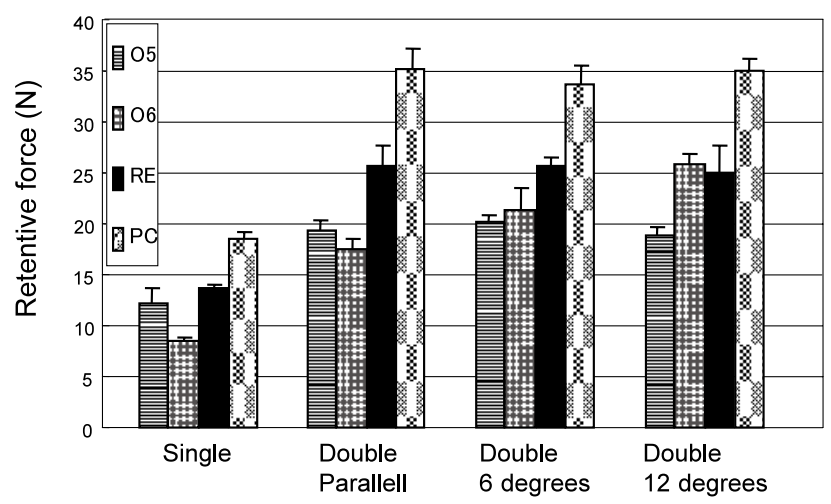

Fig. 8 Retentive force of a double retainer with three angles. O5: O-ring 2.8-mm diam., O6: O-ring 3.8-mm diam., $\mathrm{RE}$ : Autopolymerized acrylic resin, PC: Protection cap.

ble retainer; it was approximately 1.5 to 2 times higher than the retentive force of the single retainer. There were no significant differences between the three angles $(0,6,12$ degrees) of the two ND4.3 implants $(P<0.05)$. Figure 9 compares the different contact areas between the patrix and matrix. The retentive force obtained by the full contact between the patrix and matrix was approximately two times greater than that obtained with upper half contact between these denture components $(P<0.05)$.

\section{Discussion}

This study was performed to measure the retentive force of a new attachment system designed for ND-retained overdentures since there are no manufacturer's recommendations for appropriate retention systems. ${ }^{10-12}$ As for the retentive force of OPA attachments for root-retained overdentures, four sizes of O-rings (\#1 to \#4, Inoue attachment) have been used as the matrix for an OPA system (Inoue attachment). ${ }^{17-19}$ In this system, plastic patterns for post anchors with $0.21 \mathrm{~mm}$ to 0.30 $\mathrm{mm}$ undercuts are cast for the patrix. Regarding the retentive force of an OPA attachment, Suzu$\mathrm{ki}^{18}$ and Suzuki et $\mathrm{al}^{19}$ reported that the retentive force of the O-rings \#3 and \#4 was $4.75 \mathrm{~N}$ and $5.29 \mathrm{~N}$, respectively, under similar conditions. Although the retentive force of the O-ring-ND3.0 system (O3) in this study was greater than that for the OPA system (\#3), the retentive force of O4 was nearly the same as that of the OPA system. The retentive force of the OPA system was produced by the elasticity of the O-ring, depend-

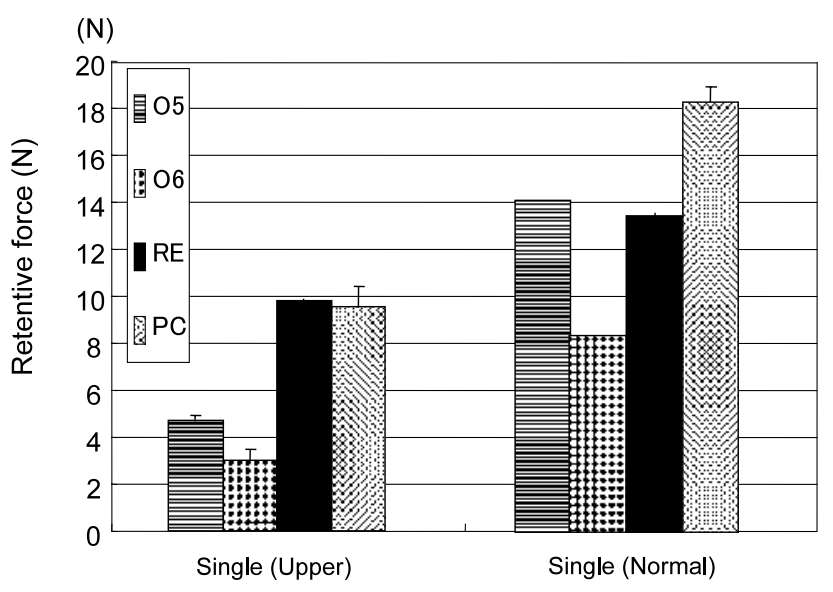

Fig. 9 Comparison of the different contact areas between the patrix and matrix. O5: O-ring, 2.8-mm diam., O6: O-ring, 3.8-mm diam., RE: Autopolymerized acrylic resin, PC: Protection cap.

ing on the amount of undercut of the patrix. ${ }^{19}$ However, the retention of ND occurred due to the friction between the O-ring and the abutment head. ${ }^{11-16}$ The smaller O-ring created greater friction and provided greater retentive force, whereas the O5-ND3.0 system was too large, and there was little friction. In addition, the abutment head of the ND4.3 retainer has six degrees of taper. The differences in retentive force depend on the position of the O-ring on the abutment head. The retentive force decreases when the O-ring is placed higher than when it is placed at a lower position on the abutment head. Furthermore, contact between only the upper half of $\mathrm{RE}$ and $\mathrm{HC}$ also produced less retentive force than that produced by the full contact between RE and $\mathrm{HC}$. Therefore, the use of a higher abutment head of ND exposed from the gingivae is recommended if there is sufficient denture space.

Since the abutment head of ND is conicalshaped with six degrees of taper, it is frequently used as an inner crown for Konus telescopes or coping telescopes. ${ }^{11,12}$ These telescopes applied to ND provided suitable support in the overdentures. An existing denture was generally immediately modified to an ND-retained overdenture by fitting autopolymerized PMMA between the ND head and the denture base by using the overlay technique as a coping telescope system after the ND was placed. ${ }^{11}$ Shirato ${ }^{20}$ reported that the retentive force of coping telescopes fabricated by a similar method in this study was $16 \mathrm{~N}$ to $23 \mathrm{~N}$ for flat-topped surfaces on inner crowns with 8 
degrees of taper, a height of $6 \mathrm{~mm}$, and a diameter of $8 \mathrm{~mm}$. In this study, the retentive force of coping telescopes was found to be less because the taper and size were different. Note that the retentive force of the telescope system increased as the masticatory force applied on the outer crown fit to the inner crown increased. Since the ND head has three very shallow grooves without an undercut, mechanical retention may be added to conventional frictional retention.

When there are more than two retainers in the same jaw, the total retention of the removable prosthesis is generally affected by the parallel adjustment of the retainers. In many cases, the strict parallelism of ND placement cannot be obtained without a surgical template fixed to the bone by a Guided Anchor Pin. In cases of nonparallel placement, the abutment head of ND can be cut with a carbide bur to achieve parallel placement. ${ }^{11}$ However, in cases where the placement of ND is slightly nonparallel without cutting, the total retention of the ND-retained overdentures is changed compared to cases in which the placement of ND is parallel.

There were no significant changes in the retentive force of the two OPA attachments with angles ranging from 0 to 20 degrees, as reported by Suzuki. ${ }^{18,19}$ In addition, the double OPA had a retentive force that was approximately two times higher than that of the single OPA. In this study, the angles of ND also did not affect the total retentive force of the double retainer. Moreover, the retentive force of the ND double retainers was approximately 1.5 to 2 times higher than that of the single ND retainers. The total retentive force can thus be estimated by the number of NDs used.

The degree of the best retentive force of a retainer is not recognized for removable partial dentures or overdentures. Generally, one removable prosthesis may require approximately 19.6 to $29.4 \mathrm{~N}$ so that it is not removed when sticky foods are chewed and so that patients can remove it easily. However, little is known about the amount of harmful force required to pull out an implant, particularly when ND is immediately loaded just after placement. ${ }^{11}$ In cases of immediate loading of ND-retained overdentures, less than $19.6 \mathrm{~N}$ of retentive force might be sufficient. Thus, a force of less than $19.6 \mathrm{~N}$ can be divided by the number of NDs. If four NDs are used, less than $4.9 \mathrm{~N}$ is recommended as a suitable retentive force; likewise, less than $6.86 \mathrm{~N}$ is suggested for three NDs; the use of $\mathrm{O} 5$ or RE is recommended for two ND4.3 retention systems.

By using ND with an O-ring, the stability and comfort of the existing complete dentures could be immediately improved. Additionally, lateral harmful force to ND would be decreased by the elasticity of the $\mathrm{O}$-ring. If the retentive force is decreased, the O-ring can be easily replaced with a new one. Although ND3.0 and ND4.3 were tested in this study, other NDs (e.g., ND3.5 diam., ND 5.0 diam., ND Groovy, ND Oval, ND Posterior) are commercially available. Further studies are necessary if the other NDs are to be used for implant-retained overdentures. Studies are also needed to clarify the change in retentive force after long-term use.

\section{Conclusion}

To evaluate the new retention system for ND-retained overdentures, four sizes of rubber $\mathrm{O}$-rings were fitted to ND abutment heads, and the retentive force was measured. Based on the results of the present experiment, it can be concluded that ND with a medium-sized O-ring, PMMA, and an ND healing cap provided appropriate retentive force. When the smallest O-ring (O3) and healing caps (HC, PC) were used for ND3.0 and 4.3 , respectively, the greatest retentive force was obtained for ND implant-retained overdentures. The retentive force of $\mathrm{O} 3$ was approximately 2.8 times greater than that for $\mathrm{O} 4, \mathrm{RE}$, and $\mathrm{HC}$. Under the angle of 12 degrees created by two ND4.3 implants, the double retainer with its various angles provided equivalent retentive force. In all the materials for ND 4.3, a greater retentive force resulted from the use of higher abutment heads.

Acknowledgement: The author greatly appreciates Professor Toshio Hosoi, Director of Department of Removable Prosthodontics, Tsurumi University School of Dental Medicine, for his helpful advice on this paper. The author also wishes to thank Dr. Chikahiro Ohkubo, Assistant Professor who always gave valuable advice.

Editing assistance of Mrs. Jeanne Santa Cruz, Baylor College of Dentistry, TX, USA, is much appreciated. This study was partially supported by a research grant from Tsurumi University Dental Society Foundation (Project No. 16005). 


\section{References}

1. Schnitman PA, Wohrle PS, Rubenstein JE. Immediate fixed interim prostheses supported by twostage threaded implants: methodology and results. J Oral Implantol 16: 96-105, 1990.

2. Chiapasco M. Early and immediate restoration and loading of implants in completely edentulous patients. Int J Oral Maxillofac Implants 19: 76-91, 2004.

3. Engquist B et al. Simplified methods of implant treatment in the edentulous lower jaw. Part II: Early loading. Clin Implant Dent Relat Res 6: 90-100, 2004.

4. Chiapasco M, Gatti C. Implant-retained mandibular overdentures with immediate loading: a $3^{-}$to 8 -year prospective study on 328 implants. Clin Implant Dent Relat Res 5: 29-38, 2003.

5. Ostman PO, Hellman M, Sennerby L. Direct implant loading in the edentulous maxilla using a bone density-adapted surgical protocol and primary implant stability criteria for inclusion. Clin Implant Dent Relat Res 7: 60-69, 2005.

6. Popper HA, Popper MJ, Popper JP. The Branemark Novum protocol: description of the treatment procedure and a clinical pilot study of 11 cases. Int J Periodontics Restorative Dent 23: 459-465, 2003.

7. Malo P, Rangert B, Nobre M. "All-on-Four" immediate-function concept with Branemark System implants for completely edentulous mandibles: a retrospective clinical study. Clin Implant Dent Relat Res 5: 2-9, 2003.

8. Malo P, Rangert B, Nobre M. All-on-4 immediatefunction concept with Branemark System implants for completely edentulous maxillae: a 1-year retrospective clinical study. Clin Implant Dent Relat Res 7: 88-94, 2005.
9. Parel SM, Schow SR. Early clinical experience with a new one-piece implant system in single tooth sites. J Oral Maxillofac Surg 63: 2-10, 2005.

10. Hahn J. One-piece root-form implants: a return to simplicity. J Oral Implantol 31: 77-84, 2005.

11. Kobayashi M, Ohkubo C, Sato J et al. The use of 1-piece implant (NOBEL DIRECT ${ }^{\mathrm{TM}}$ ). Japanese Journal of Maxillo Facial Implants 3: 13-19, 2005.

12. Ohkubo C, Kobayashi M, Sato J. Early and immediate loading: application of one-piece implant system. Dental Outlook 107: 291-297, 2006.

13. Babbush CA. Provisional implants: surgical and prosthetic aspects. Implant Dent 10: 113-120, 2001.

14. Leshem D, Mazor Z, Leshem R et al. A simple technique for fabrication of immediate interim removable prosthesis supported by transitional implants. Implant Dent 12: 227-231, 2003.

15. Kobayashi M, Ohkubo C, Suzuki Y et al. Retentive force of O-ring attachment to use Immediate Provisional Implant (IPI)-retained overdenture. Eur J Prosthodont Restor Dent 13: 147-149, 2005.

16. Ohkubo C, Sato J, Hosoi T et al. O-ring attachments for transitional implant-retained overdentures. J Prosthet Dent 91: 195-197, 2004.

17. Ohyama T. Clinical study on the new stud attachment with O-ring. Tsurumi Univ Dent J 2: 91-110, 1976.

18. Suzuki Y. Experimental studies on the retentive force of OPA-attachment. Tsurumi Univ Dent J 8: 13-33, 1982.

19. Suzuki Y, Miyata T, Obana J. OPA attachment for the maxilla and a telescopic prosthesis for the mandible. Hotetsu Rinsho 153-158, 1983.

20. Shirato K. Experimental studies on the accuracy and the retentive force of coping telescope. Tsurumi Univ Dent J 17: 131-150, 1991. 